\title{
CRITERIA FOR THE IRREDUCIBILITY OF A RECIPROCAL EQUATION.
}

BY PROFESSOR L. E. DICKSON.

(Read before the Chicago Section of the American Mathematical Society, April 17, 1908.)

1. A reciprocal equation $f(x)=x^{m}+\cdots=0$ is one for which

$$
x^{m} f(1 / x) \equiv c f(x) .
$$

Replacing $x$ by $1 / x$, we see that $f \equiv c^{2} f, c= \pm 1$. Now $f(x)$ has the factor $x \pm 1$ and hence is reducible, unless $m$ is even and $c=+1$. Further discussion may therefore be limited to equations

(1) $F(x) \equiv x^{2 n}+c_{1} x^{2 n-1}+c_{2} x^{2 n-2}+\cdots+c_{2} x^{2}+c_{1} x+1=0$

of even degree and having

$$
x^{2 n} F(1 / x) \equiv F(x) .
$$

Let $R$ be a domain of rationality containing the $c$ 's.

Under the substitution

$$
x+1 / x=y,
$$

$x^{-n} F^{\prime}(x)$ becomes a polynomial in $y$,

$$
\phi(y)=y^{n}+k_{1} y^{n-1}+\cdots+k_{n},
$$

with coefficients in $R$. By a suitable choice of the $c^{\prime} s$, the $k$ 's may be made equal to any assigned values.

We shall establish in $\S \S 2-7$ the following:

TheOREm. Necessary and sufficient conditions for the irreducibility of $F(x)$ in the domain $R$ are

(I) $\phi(y)$ must be irreducible in $R$.

(II) $F(x)$ must not equal a product of two distinct irreducible functions of degree $n$.

The second condition is discussed in $\S \S 8-10$.

2. The irreducibility of $F(x)$ in $R$ implies that of $\phi(y)$. For, if 


$$
\phi(y)=\left(y^{l}+\cdots\right)\left(y^{m}+\cdots\right), \quad(l+m=n, \quad l>0, \quad m>0),
$$

then would

$$
F(x)=x^{l}\left\{(x+1 / x)^{l}+\cdots\right\} \cdot x^{m}\left\{(x+1 / x)^{m}+\cdots\right\} \cdot
$$

3. If $F(x)$ has in $R$ an irreducible factor

$$
A(x)=x^{2 r+1}+a_{1} x^{2 r}+\cdots+a_{2 r} x+a_{2 r+1}
$$

of odd degree, then $F(x)$ has the irreducible factor.

$$
B(x) \equiv \frac{x^{2 r+1}}{a_{2 r+1}} A\left(\frac{1}{x}\right)=x^{2 r+1}+\frac{a_{2 r}}{a_{2 r+1}} x^{2 r}+\cdots+\frac{a_{1}}{a_{2 r+1}} x+\frac{1}{a_{2 r+1}},
$$

not identical with $A(x)$. For, from $F \equiv A Q$ and (2) follows

$$
F(x) \equiv B(x) Q^{\prime}, \quad Q^{\prime} \equiv a_{2 r+1} x^{2 n-2 r-1} Q(1 / x) .
$$

Next, if $B \equiv A$, then

$$
\begin{aligned}
A=x^{2 r+1} \pm 1 & +a_{1} x\left(x^{2 r-1} \pm 1\right) \\
& +a_{2} x^{2}\left(x^{2 r-3} \pm 1\right)+\cdots+a_{r} x^{r}(x \pm 1),
\end{aligned}
$$

so that $A$ would have the factor $x \pm 1$ and be reducible.

4. If $\phi(y)$ is irreducible in $R, F(x)$ has in $R$ no irreducible factor $A$ of odd degree $<n$. For, if so, $P \equiv A B$, where $B$ is given in $\S 3$, would be a self-reciprocal factor of $F(x)$. In fact,

$$
P(1 / x)=A(1 / x) A(x) / x^{2 r+1} a_{2 r+1}, \quad x^{2(2 r+1)} P(1 / x)=A B=P(x) .
$$

Hence, in view of $(3), x^{-(2 r+1)} P(x)$ would equal a factor of degree $2 r+1$ of $\phi(y)$.

5. If $F(x)$ has in $R$ an irreducible factor

$$
A(x)=x^{2 r}+a_{1} x^{2 r-1}+\cdots+a_{2 r-1} x+a_{2 r}
$$

of even degree, then $F(x)$ has the irreducible factor

$$
B(x)=\frac{1}{a_{2 r}} x^{2 r} A\left(\frac{1}{x}\right) .
$$

If $B(x) \equiv A(x), A$ is self-reciprocal, viz., $A(x)=x^{2 r}+1+a_{1}\left(x^{2 r-1}+x\right)+\cdots+a_{r-1}\left(x^{r+1}+x^{r-1}\right)+a_{r} x^{r}$. 
In fact, the conditions for $B \equiv A$ are

$$
a_{2 r}= \pm 1, \quad a_{2 r-1}= \pm a_{1}, \quad a_{2 r-2}= \pm a_{2}, \cdots .
$$

For the lower signs, $A$ has the factor $x^{2}-1$, contrary to hypothesis.

6. If $\phi(y)$ is irreducible in $R, F(x)$ has in $R$ no irreducible factor $A$ of even degree $<n$. For, by $\S 5$, either $B$ is distinct from $A$ so that $A B$ is a self-reciprocal factor of $F(x)$, or else $A$ itself is a self-reciprocal factor. In either case $\phi(y)$ would have in $R$ a factor of degree $<n$.

7. It follows from $\S \S 4,6$ that, when $\phi(y)$ is irreducible in $R, F(x)$ has no irreducible factor of degree $<n$. Further, by $\S \S 3,5$, an irreducible factor $A(x)$ of degree $n$ implies a second irreducible factor $x^{n} A(1 / x)$, algebraically distinct from $A(x)$. The theorem of $\S 1$ is therefore proved.

8. It remains to consider the case $F=A B$,

$$
\begin{aligned}
& A=x^{n}+a_{1} x^{n-1}+\cdots+a_{n}, \\
& B=x^{n}+\frac{a_{n-1}}{a_{n}} x^{n-1}+\cdots+\frac{a_{1}}{a_{n}} x+\frac{1}{a_{n}},
\end{aligned}
$$

where $A$ and $B$ are distinct irreducible functions in $R$. To determine the $a_{i}$, we have $n$ distinct relations

$$
a_{1}+a_{n-1} / a_{n}=c_{1}, \quad a_{2}+\left(a_{1} a_{n-1}+a_{n-2}\right) / a_{n}=c_{2}, \cdots
$$

We may eliminate $a_{1}, \cdots, a_{n-1}$ and obtain an equation for $a_{n}$. As shown in $\S 9$, this equation is of degree $2^{n}$. Except for certain sets of values of the $c_{i}$, we may express $a_{1}, \cdots, a_{n-1}$ rationally in terms of $a_{n}$; the problem is then reduced to the consideration of the rationality of a root of the equation of degree $2^{n}$. This equation for $a_{n}$ is a reciprocal equation. In fact, if we set

$$
A_{1}=a_{n-1} / a_{n}, \quad \cdots, \quad A_{n-1}=a_{1} / a_{n}, \quad A_{n}=1 / a_{n},
$$

equations (5) become

$$
\text { (5') } A_{1}+A_{n-1} / A_{n}=c_{1}, A_{2}+\left(A_{1} A_{n-1}+A_{n-2}\right) / A_{n}=c_{2}, \cdots \text {. }
$$

That the equations $\left(5^{\prime}\right)$ are throughout of the same form as equations (5) is evident from the fact that we have merely interchanged the rôles of the factors $A$ and $B$ of $F$. Hence the equation in $a_{n}$, obtained by eliminating $a_{1}, \cdots, a_{n-1}$ from 
(5), is identical with the equation in $A_{n}=1 / a_{n}$, obtained from $\left(5^{\prime}\right)$.

9. Denote the roots of $F=0$ by

$$
\alpha_{1}, \quad \alpha_{1}^{-1}, \quad \alpha_{2}, \quad \alpha_{2}^{-1}, \quad \cdots, \quad \alpha_{n}, \quad \alpha_{n}^{-1} .
$$

A factorization $F=A B$, of the kind considered in $\S 8$, corresponds uniquely to a separation of the roots (6) into two sets each of $n$ roots, such that reciprocal roots belong to different sets. Hence the roots of the first set may be selected in

$$
\frac{2 n(2 n-2)(2 n-4) \cdots 2}{n !}=2^{n}
$$

ways. The number of factors $A$ is thus $2^{n}$.

10. For $n=2$, we set $\alpha_{1}=\alpha, \alpha_{2}=\beta$, and have

(7) $\alpha+\beta+\alpha^{-1}+\beta^{-1}=c_{1}, 2+\alpha \beta^{-1}+\beta \alpha^{-1}+\alpha \beta+\alpha^{-1} \beta^{-1}=c_{2}$.

From these we derive

$$
\alpha^{2}+\beta^{2}+\alpha^{-2}+\beta^{-2}=c_{1}^{2}-2 c_{2} .
$$

Hence $\alpha \beta+\alpha^{-1} \beta^{-1}$ and $\alpha \beta^{-1}+\alpha^{-1} \beta$ are the roots of

$$
z^{2}-\left(c_{2}-2\right) z+c_{1}^{2}-2 c_{2}=0 .
$$

The quartic for $a_{2}(\S 8)$ is obtained by setting

$$
z=a_{2}+a_{2}^{-1} \text {. }
$$

By $\left(7_{1}\right), \alpha+\beta$ is a rational function of $\alpha \beta$ and $c_{1}$ when $c_{1} \neq 0$, Hence, for $c_{1} \neq 0$, the necessary and sufficient conditions for the factorization $F=A B$ in $R$ are that the roots

$$
z_{ \pm}=\frac{1}{2}\left(c_{2}-2\right) \pm\left[\left(1+\frac{1}{2} c_{2}\right)^{2}-c_{1}^{2}\right]^{1 / 2}
$$

of (8) be rational and that one of the values $\left(z_{ \pm}^{2}-4\right)^{1 / 2}$ be rational, so that (9) shall lead to a rational value of $a_{2}$. Incorporating the condition that (4) shall be irreducible in $R$, we obtain the

THeOrem.* The necessary and sufficient conditions that

$$
x^{4}+c_{1} x^{3}+c_{2} x^{2}+c_{1} x+1 \quad\left(c_{1} \neq 0\right)
$$

* For other proofs by the writer, see Amer. Math. Monthly, vol. 10 (1903), p. 221 ; vol. 15 (1908), p. 75 . The first paper cited also treats reciprocal sextic equations. 
shall be irreducible in a domain $R$ are that $\left(c_{1}^{2}-4 c_{2}+8\right)^{1 / 2}$ be irrational, and that either $l=\left[\left(1+\frac{1}{2} c_{2}\right)^{2}-c_{1}^{2}\right]^{1 / 2}$ be irrational or else $l$ rational and $\left[\frac{1}{2} c_{2}^{2}-c_{1}^{2}-2 \pm\left(c_{2}-2\right) l\right]^{1 / 2}$ both irrational.

11. The only linear fractional transformations which replace a reciprocal equation by a reciprocal equation are

$$
x^{\prime}= \pm \frac{\alpha x+\beta}{\beta x+\alpha} \quad\left(\alpha^{2} \neq \beta^{2}\right) .
$$

Then $y$, given by (3), undergoes the transformation

$$
y^{\prime}= \pm \frac{\left(\alpha^{2}+\beta^{2}\right) y+4 \alpha \beta}{\alpha \beta y+\alpha^{2}+\beta^{2}} \text {. }
$$

The transformation on $\frac{1}{2} y$ is the square of (11).

UnIVERSITY of Chicago,

March, 1908.

\section{A NEW GRAPHICAL METHOD FOR QUATERNIONS.}

BY PROFESSOR JAMES BYRNIE SHAW.

(Read before the Southwestern Section of the American Mathematical Society, November $30,1907$. )

1. ANY quaternion $q$ may be written in the form $q=(w+x i)+(y+z i) j$. For convenience let us represent numbers of the form $w+x i$ (practically equivalent to ordinary complex numbers save in their products by $j$ ) by Greek characters, so that $q$ may be written

$$
q=\alpha+\beta j
$$

where for any number $\beta$ we have $\beta j=j \bar{\beta}, \bar{\beta}$ being the conjugate of $\beta$.

The tensor of $q$ is then the square root of the sum of the squares of the moduli of $\alpha, \beta$. Also the scalar of $q$ is $\frac{1}{2}(\alpha+\bar{x})$, that is, the real part of $\alpha$.

2. The product of $q=\alpha+\beta j$ and $r=\gamma+\delta j$ is

and also we have

$$
q r=(\alpha \gamma-\beta \bar{\delta})+(\alpha \delta+\beta \bar{\gamma}) j,
$$

$$
r q=(\alpha \gamma-\overline{\beta \delta})+(\bar{\alpha} \delta+\beta \gamma) j .
$$

